\title{
What should we know to develop an information robot?
}

Satoru Satake, Keita Nakatani, Kotaro Hayashi, Takyuki Kanda, Michita Imai

This paper is aimed at identifying the required knowledge for information robots. We addressed two aspects of this knowledge, 'what should it know' and 'what should it do'. The first part of this study was devoted to the former aspect. We investigated what information staff know and what people expect from information robots. We found that there are a lot of similarities. Based on this, we developed a knowledge structure about an environment to be used to provide information. The developed knowledge structure worked well. In the field study we confirmed that the robot was able to answer most of the requests (96.6\%). However, regarding the latter aspect, although we initially replicated what human staff members do, the robot did not serve well. Many users hesitated to speak, and remained quiet. Here, we found that knowledge for facilitating interaction is missing. We further designed the interaction flow to accommodate people who tend to be quiet. Finally, our field study revealed that the improved interaction flow increased the success ratio of information providing from $54.4 \%$ to $84.5 \%$. 


\section{What Should We Know to Develop an Information 3 Robot?}

4 Satoru Satake, Keita Nakatani, Kotaro Hayashi, Takayuki Kanda, Michita Imai

5 ATR

$6 \quad 090-7258-7247$

7 Fax: 099-7258-7247

8 satoru@atr.jp

Abstract This paper is aimed at identifying the required knowledge for information robots. We addressed two

12 the former aspect. We investigated what information staff know and what people expect from information robots.

13 We found that there are a lot of similarities. Based on this, we developed a knowledge structure about an

14 environment to be used to provide information. The developed knowledge structure worked well. In the field study

15 we confirmed that the robot was able to answer most of the requests (96.6\%). However, regarding the latter aspect,

16 although we initially replicated what human staff members do, the robot did not serve well. Many users hesitated to

17 speak, and remained quiet. Here, we found that knowledge for facilitating interaction is missing. We further

18 designed the interaction flow to accommodate people who tend to be quiet. Finally, our field study revealed that the

19 improved interaction flow increased the success ratio of information providing from $54.4 \%$ to $84.5 \%$.

20 Keywords Information-providing, Direction-giving, Belief about robots

\section{Introduction}

23 Direction-giving is often considered as a desired task for social robots and embodied agents [124 4]. In our daily life, one of the roles that frequently offer direction-giving is information service 25 (Figure 1). Such information booth/counter can be found in stations, airports, shopping malls, 26 and sightseeing places.

29 Satoru Satake satoru@atr.jp,Keita Nakatani k-nakatani@atr.jp, Kotaro Hayashi, 30 hayashik@atr.jp, Takayuki Kanda kanda@atr.jp, Michita Imai michita@ailab.ics.keio.ac.jp 
31 We wonder what would be the required 'knowledge' to develop a robot that engages in such an 32 information service. Probably, most of us have experience using information service, and many 33 of us believe that we know what the information service is. Thus, one would argue that it is just 34 easy to develop such a robot. One might say that "I know from common sense what the 35 information service is. I can just implement it." Is this true?

We started the study with two research questions:

- Is our common knowledge about the tasks of information service (i.e. what they serve) applicable to information robot?

- Can we create an information robot by replicating what human information staff knows and does? Or, is there any missing knowledge?

We first investigated what people would expect from an information robot, and confirmed that there are a lot of similarities with what human information staff does (section 3 ). Thus, we decided to use knowledge about human information staff (what they know and what they do), and developed an information robot (section 4). However, about the second research question, the assumption was not true. Thus, we further investigated missing knowledge (section 5 and 6).

\section{RELATED WORKS}

\subsection{Information-Providing Robots}

Robots have been deployed as tour guides. There were a couple of museum robots that navigated around the environment and provided explanations [5]. Robots are also used for interactive information-providing. For instance, Gross et al., developed an article search robot, which enables visitors to request an item and let the robot navigate to its location. [6]. Input for these robots is often with GUIs, thus there are lists of destinations / items, from which users choose one.

In contrast, in case of dialog-based system, the difficulty is to predict the set of requests users could ask. Thus, there are assumptions made for the input, like name of locations. For instance, a virtual agent, Mack, developed by Cassell et al., is able to respond the name of locations and people in office, and provide direction-giving [1] [2]. But, in a real-world natural interaction, what users would ask is not bounded by such assumptions. In [7], the robot provided 
60 direction-giving interaction in response to the name of locations in a shopping mall, and

61 exceptions were handled by a human operator. That is, the system yet did not by itself address

62 the questions beyond the assumptions.

63 Overall, in the previous studies, it was little explored what people would ask/request in

64 information dialog with a robot. In contrast, we found that people ask various requests beyond

65 name of locations, and identified a required knowledge representation.

66

67

68

69

70

\subsection{Direction-giving Interaction}

It is reported that a good direction consists of pairs of actions and landmarks [8], such as "turn right at the post office, and ...". To provide such explanations, there is a technique to build a knowledge about spatial relationships among shops and corridors [9]. There are techniques to make a robot understand directions from humans [10]; in their study, the representation stores the relationship between description of the entities in the space and the map.

In these studies, the common assumption is that a system is able to provide directions if the name of a location is asked. In contrast, our study reveals other type of requests in information dialog, and we report on the needed knowledge representation.

Note that it is well known in HRI studies that gaze and pointing gesture make the interaction more natural and effective (e.g. [11, 12]). The use of gesture in direction-giving is also studied in conversational agent [2] as well as in human-like robots [3, 4]. Our directiongiving behavior is informed by these studies.

\subsection{Engagement}

In our study, we noticed some visitors remained silent, even after directly approaching the robot, and hearing its requests to engage. Relevant to this, there were studies about "engagement" process, that is, when people participate and feel connected in collaboration, their gaze well meet with each other and they do not quit the interaction [13]. Rich and his colleagues developed a technique to detect engagement using people gaze [14]. Kobayashi and his colleagues developed a technique to select a person to whom a robot should ask questions in multi-party interaction, in the way a teacher appoints a student in a class for an answer [15]. Their technique is based on the findings that people nodding and engaging in mutual gaze are more likely to answer than someone avoiding meeting gaze. In contrast, in our study, the silent visitors were people who 
89 voluntarily approached the robot. They typically behaved as if they were willing to interact with

90 the robot but did not talk with it.

\section{$91 \quad 2.4$ Knowledge Representation}

92 There are several computer applications e.g. Google search or Apple's Siri that provides

93 information related to locations. There are many similar aspects between our robot and such

94 applications, e.g. both need connection between language and local knowledge, interpretation

95 needs to be contextual, and answers to be provided in verbal way. Thus, similarly to these

96 approaches, we used ontology [16] to build the knowledge representation. However, we need to

97 build our own knowledge representation because required knowledge structure is different, and

98 we cannot simply apply existing software like Google search and Siri for the robot. For instance,

99 robots can use pointing gesture (also, often robots are not equipped with display), which very

100 much change the way of giving direction.

101

102

\section{INFORMATION IN A SHOPPING MALL}

103 We investigated the daily tasks of information service employees and what visitors typically

104 expect from robots acting as such. We found a lot of similarities. The study protocol was

105 approved by institutional review boards of Advanced Telecommunications Research Instituted

106 International with reference number 14-502-2.

\section{3.1 Daily Tasks of Information Service}

108 We interviewed two employees working at the information desk of a shopping mall (Figure 1, 109 left).

110 First we asked an overall description of their job: they usually wait for visitors to come at

111 the information booth. They were requested by the mall administrators to serve as 'information

112 staff'. Only procedures for lost items were provided. For other tasks (e.g. information providing)

113 they use their common sense.

114 Further, we asked them to categorize the typical requests from visitors, and how they would 115 respond. Both reported that there are three types of requests: 
116 Direction giving: They reported that this is the most frequent request. Visitors ask simple 117 where-type questions, e.g. "where are the toilets?" In addition to the name of locations, people 118 use other popular name, like "hello show", or the name of designated areas, like "smoking area".

119 Their typical response is to provide turn-by-turn directions using utterance and pointing gesture.

120 When visitors do not understand, they sometimes write down to a map, or rarely they take them 121 to the destination.

122 Recommendation (inquiry): When a visitor does not know whether there are shops that 123 meet his needs, he may ask it to the information staff. Visitors may inquire characteristics of

124 shops, such as name of items, and category of shops. Here are some examples of questions: "Are 125 there Japanese restaurants?" "Are there shops that sell Osaka souvenirs?” The staff members 126 typically verbally list the shops or events that meet their criteria. Visitors sometimes ask for a 127 recommendation from the staff without providing solid conditions but only with subjective 128 words e.g. "Are there any good restaurants?" For such request, the staff members reported that 129 they typically try not to give a subjective preference, because their preferences may or may not 130 match with visitors'. Thus, their response for inquiry and recommendation are similar: they try to 131 objectively reply and provide a list of shops that seem appropriate.

132 Lost child and lost-and-found: When children are lost, or they lost some items, they come 133 to the information desk. For lost child, the staff usually makes a public announcement throughout 134 the shopping mall. Lost items can be retrieved at the information booth when available upon 135 confirmation of the owner.

\section{$136 \quad 3.2$ Expectations from Information Robot}

137 To investigate what people expect from information robot, we interviewed customers in the 138 shopping mall. To find people who are willing to help us collecting knowledge for future robots, 139 we prepared a situation where visitors can see a robot in interaction. Thus, we prepared a robot 140 for information, which is controlled with wizard-of-oz method. Then, we asked people who 141 stopped around the robot and/or interacted with it to participate to the interview. 21 visitors 142 participated.

143 In the interview, we asked the visitors to imagine future situation where robots will be 144 capable to offer information service as they like, regardless of the robot's capability they 
145 observed previously. Then, we asked them to freely provide as many functions they would like 146 information robots to have.

147 The interviews were recorded, and transcribed for analysis. We categorized the different

148 kind of requests expressed by the visitors, For instance, visitors reported sentences such as,

149 "I often look for the smoking area, thus I would like to ask the robot about it."

150 This utterance was coded as expectation for direction giving, because we interpret it as

151 'where'-type question in which visitors simply want to know the location. The followings ones

152 were coded as expectation for recommendation (inquiry):

153 "I'd like to know about sports and furniture shops"

154 "The shop which sells the most? Well, I want the robot give me recommendations of shops"

155 Such cases were classified as recommendation (inquiry), because visitors need to know more 156 information than just a location.

157 Then, two coders who do not know the research purpose judged whether each transcribed 158 sentence would fit into the above defined categories, or not (which is categorized as 'other').

159 Two coders' judgment matches reasonably well yielding kappa coefficient .857 .

Table 1 shows the coding result. The ratio of visitors who mention the expectation is listed in each row. They can provide multiple answers, thus the sum of the ratios exceeds $100 \%$.

The visitors' expectation for the information robot largely overlaps with what human information daily do. Almost all visitors (20 out of 21) mentioned that they expect direction giving and the majority (16 out of 20) reported that they expect the robot to offer turn-by-turn direction accompanied with pointing gesture. For instance, one spontaneously mentioned the practicality of pointing gesture in directions giving: useful if the robot could do pointing gestures," wanted the robot to explain with a map. 
176

177

178

179

180

181

182

183

184

185

186

187

188

189

190

191

192

193

194

195

196

197

198

199

200

201

202

203

204

205

"I'd like to know what kind of shop it is, its atmosphere, what it sells, and so on."

In contrast, the 'playing with children' category, is specific to the information robot. We collected comments such as:

"Interacting with the robot was enjoyable. This is good for people who come with their children"

"Many families only have one child. It would be nice if the robot behaved like a brother"

\subsection{Requirements}

People's expectation toward information robots largely overlapped with what is served at human information. That is, most of them expect two services: direction giving and recommendations. Thus, in this study, we focused on these two services.

Further we investigated the required knowledge to be stored. We analyzed the utterances of the requests. We labeled them based on the type of request. For instance, we assigned a label 'name of location' to the utterance "I'd like to know where the event dream world takes place", 'name of item' to the utterance "I'd like to know where can I buy coffee". If multiple labels are applicable we assigned all of them. Labels are merged when possible, resulting in 6 different labels. To confirm the classification, we asked two coders who do not know the research purpose to classify the utterances based on the 6 labels. Their coding matches reasonably well, yielding kappa coefficient of .637.

Finally, we identified that the following information is needed:

1) Name of location: such as names of shops or names of events. In addition to the formal name, people use various nicknames. $78.3 \%$ of people mentioned this category.

2) Item name: people look for specific product or entity available in shops. For instance, this category includes items such as "cell phone charger" and "coffee". $47.8 \%$ of people mentioned this category.

3) Category: shops can usually be grouped into larger categories, like "restaurant", "Japanese restaurant". $52.2 \%$ of people mentioned this category.

4) Features: shops are usually recognized as some generally-known features, like "good view", "expensive", and "recommended." $65.2 \%$ of people mentioned this category.

5) People activity: locations are sometimes referred as the activity that people do there, like "play", "eat", "shop". $60.9 \%$ of people mentioned this category. 
206

214 robot.

\section{System}

\subsection{Architecture}

217 Our goal is to develop a robot that autonomously provides information service. For this purpose,

218 based on the analysis in section 3, we developed a knowledge representation that can be used by

219 such robot. Figure 2 shows the architecture of the system. Information from sensors goes through

220 modules like people tracking (explained in section 4.5.2), localization (section 4.5.3), and speech

221 recognition (section 4.5.4). Output from these modules are used in the behavior controller

222 (section 4.3), which contains a dialog manager (section 4.4). The environmental knowledge is 223 stored in ontology (section 4.2.2) and map (section 4.2.3), and used by the dialog manager. We 224 explain these modules in the later section.

\subsection{Knowledge representation}

227 There are two types of information in the knowledge representation. One is the map used for 228 direction giving (explained in section 4.2.3). The other is shop-related data (explained in section 229 4.2.2).

\subsubsection{Environment}

231 The study was conducted in a big shopping mall located in a suburban area. It consists of three

232 buildings (Figure 3 left), one having 12 floors, and others having 6 floors. There are 51 shops, 31 
233 restaurants, 42 facilities, 6 event halls, 4 squares (e.g. Figure 3 right), 2 stages, and many offices.

234 The mall is mainly busy during weekends. Almost all shops are for non-daily goods, like clothes, 235 shoes, sports, outdoor activities. We often observe people who look for shops and locations (e.g. 236 they look at the floor maps, and/or ask the service staff). The main hall where big events take 237 place is located far (it takes 5 minutes of walk) from the square where we put the robot, thus 238 people often ask where an event is.

\subsubsection{Ontology of entities in the map}

We designed our knowledge representation for 'request' and 'shops' together using an ontology language, OWL [16]. Figure 4 shows the designed knowledge structure, i.e. ontology. The basic element in OWL is the 'class', which has 'properties' that store the information. There are two primary classes, 'location entity' and 'requestable property' prepared.

\section{Location Entity}

We define entities like shops, facilities and events as instances of the 'location entity' class. There are three properties:

1) Name: we stored the official or commonly used name.

2) Nicknames: some shops are referred to with a nickname. We listed such nicknames people could use. For example, "Kentucky Fried Chicken" is referred as "KFC."

location. When multiple locations are available, people would prefer to select one. For instance, better, cheap, popular, etc. We store one extra property, 'introduction property,' in selective location, to be used in dialog to help people selecting locations. In contrast, people would usually 261 location class.

\section{Requestable property}


There are six types of information communicated in information dialog (section 3.3).

264

265

266

267

268

269

270

271

272

273

274

275

276

277

278

279

280

281

282

283

284

\section{5}

286

287

288

289

290

291

Except for name of location, they are realized as 'requestable property' class, which has

subclasses 'item name', 'category', 'features', 'people activity', and 'people's state'. When a user requests some information, it is turned into an instance of the 'requestable property'. Then, the location(s) having the same property will be searched. Each property item has wordings that are expected to be used in people's utterance. For instance, 'eat' (instance of people's activity subclass) is associated with wordings such as "eat", "have lunch", and "have a meal". Note that more complex requests (e.g. "Japanese" restaurant with a "good view") can be represented as multiple instances combined with 'and/or' operators, but we did not implement such complex operations because users rarely made such complex requests.

\section{Relationships between 'location entity' and 'requestable property'}

Table 2 shows possible relationships between two subclasses. For instance, some visitors could request a restaurant where they can have "pasta". To handle such requests, a "pasta" entity is prepared as an instance of 'item name' subclass which is associated with shops with the relation 'is served at". Such relation is defined inside dialog management (section 4.3) as well. Note that an instance of 'requestable property' can be associated with multiple 'location entities' (e.g. "pasta" can be served at multiple restaurants).

Finally, we prepared the data for the shopping mall (section 4.2.1). There are 201 location entities (84 shops, 75 service facilities, 39 events, and 3 buildings) with in total 3345 nicknames. There are 530 requestable properties (501 items, 163 categories, 44 features, 63 people activities, 22 people's states) prepared as well.

\subsubsection{Route Perspective Map}

Informed by [9], we manually prepared a route perspective map (illustrated in fig. 5), which consists of pairs of landmarks and actions. Using the map, the system generates turn-by-turn directions giving, such as "go straight, turn left at the book store, go out the door with exit sign, ..." The map includes the following information:

1) Topological map: Nodes are located at decision points in the map. Transition through corridor or between different floors, such as stairs, escalators, and elevators are 
292

expressed as movements between nodes. Entrances of shops, facilities, and events (i.e. location entity) are also represented as nodes.

2) Landmarks: If available, visible landmarks are manually associated for each route as denoted in [9], e.g. famous shop names with salient signboards, elevators, and escalators.

3) Actions: In [9], actions were only turning behaviors, which were computed from a topological map. In contrast, as there are many floors and multiple buildings, we added actions like "enter the next building", "go to the 3rd floor".

\subsection{Behavior Controller}

When a person stops by the robot (within $2.5 \mathrm{~m}$ for 3.0 seconds), or is detected as approaching at $2.5 \mathrm{~m}$ from it, it starts a dialog. The robot orients its body and gaze to the user. When there is no user, the robot shows liveliness by slightly moving head and arms.

During the dialog, its head and body is oriented toward the user, except for the moment when it performs a pointing gesture which is often used when giving directions. When it points at a direction, its head direction is oriented toward the pointed direction for the first three seconds of pointing in order to draw the user's attention toward the pointed direction. The robot ends the dialog when the user leaves the robot's side ( $3 \mathrm{~m}$ away), or when the dialog management module decides to end the dialog.

\subsection{Dialog Manager}

We developed a rule-based mechanism for dialog management. Assuming that there is an input coming from the speech recognition module (explained in 4.5.4), the input is turned into text and matched with name/nickname properties of location entities and with instances of requestable properties (explained in 4.2.2). If a requestable property matched, it is compared with location entities.

When only non-selective locations are matched, it chooses the nearest one. In case the user asked for a location with a specific name of location, there should be only one location to be matched. In these cases, the system provides direction-giving dialog, in which turn-by-turn directions to the location are generated. 
Otherwise, it initiates a recommendation dialog. It verbally lists the locations that match

322

323

324

325

326

327

328

329

330

331

332

333

334

335

336

337

338

339 (Figure 1, right).

340

341 We use a people tracking method described in [17], which provides an estimation of pedestrians'

342 locations every $33 \mathrm{~ms}$. It covers the square we used. There are 49 3-D range sensors attached on

343 the ceiling (combination of Panasonic D-Imager, ASUS Xtion, and Velodyne HDL-32E). with the requestable property instance one by one. For each location, it explains the location using the text in its introduction property. For instance, it utters "Ramen is served at a ramen restaurant named Kaika-ya. They serve a ramen with tuna soup. May I explain the directions to go there?" As human staff does, we carefully avoid telling subjective preferences, but only provided objective facts.

In addition, it reacts to the words for greeting. When an input matches with words like "hello", it returns a greeting utterance. When an input matches with leave-taking words like "bye", it returns leave-taking words and ends the dialog.

When no location is matched, the system explains that "(requested item) is not in this shopping mall. I only know about this mall".

\subsection{Other Modules}

\subsubsection{Robot}

We used a robot characterized by its human-like physical expressions. It is $120 \mathrm{~cm}$ high and 40 $\mathrm{cm}$ in diameter on a mobile platform. It has a 3-DOF head and 4-DOF arms. There are two $30 \mathrm{~m}$ range laser sensors attached. We used the robot with a maximum speed of $550 \mathrm{~mm} / \mathrm{sec}$ and 50 degree $/ \mathrm{sec}$ for rotations. The accelerations are set to $300 \mathrm{~mm} / \mathrm{sec} 2$ and 50 degree $/ \mathrm{sec} 2$. To clearly communicate its role, we put an 'information staff' sign in Japanese on the chest of the robot

\subsubsection{People Tracking}

the ceiling (combintion of Panasonic D-Imager, ASUS Xtion, and Velodyne HDL-32E).

\subsubsection{Localization}

345 For robot localization, we use a particle filter with a ray tracing approach on a grid map [18]. The

346 grid map is built from odometry and laser scanner data. This module is called every $350 \mathrm{msec}$ 347 and updates the robot's position. 


\subsubsection{Speech Recognition (with Human Operator)}

349 We developed fully-autonomous system using ASR (automatic speech recognition), but finally 350 to better test the overall framework we used a human operator instead of ASR.

351

352

353

354

355

356

357

358

359

360

361

362

363

364

365

366

367

368

369

370

371

372

373

374

375

376

\subsubsection{Automatic Speech Recognition (ASR)}

We used an ASR software, ATRASR [19]. It uses a language model based on FSA (Finite State Automaton). We constructed the language model mainly using the terms appeared in the ontology.

With preliminary trials using Wizard-of-oz approach, we analyzed the way visitors speak to the robot. In total, 470 requests collected over 3 days of preliminary trials. From the analysis of the requests, we found that they mainly follow three ways of speaking, as follows:

- Noun/adjectives only:

People only spoke words like a name (nickname) of location, category, or item name, such as "restaurant," and "coffee". Sometimes, for features and people's activity, they add such terms like "place for" (eat/lunch/play). Some ontology items are adjectives, such as "tired". People sometimes only spoke such adjectives.

- "Where is" question:

The above noun is used in "where is" question, such as "where is Kaika-ya (the name of restaurant)?"

- "I would like to" sentence:

People also use the form of "I would like to" + "verb" + "noun" in requesting sentences, such as "I would like to buy coffee".

For all names, nicknames, and requestable properties, we automatically generated grammatical structures for ASR. Further, we added the following grammars. First, some basic verbs like "go" can be used in "I would like to" type sentences but were not included in the ontology (as they by themselves does not represent any specific request), which we manually added ( 8 verbs). Second, we added filler words, such as "well", "ah", that appear in advance to questions (12 words). Third, to eliminate noises from environments, like sounds from people's walking, whistle from ships, we added some fillers (66 fillers). Overall, we prepared the lexicon whose size is 1469 with 4938 links. 
377

378

379

380

381

382

383

384

385

386

387

388

389

390

391

392

393

394

395

396

397

398

399

400

401

402

403

404

405

The ASR outputs the matched names, nicknames, or requestable properties, which are used in the dialog manager to determine the answer to be provided. In case the ASR detects the recognition to be less reliable (because the input does not match well with its language model), the dialog manager prompts the user to say again with utterances like "could you repeat please?" The ASR is deactivated while the robot is speaking.

We evaluated the system performance using this ASR implementation. We put the robot on a square of the mall (Fig. 3 right), and let the visitors freely use it. With our preliminary test, with 22 users, there are 81 requests, for which the robot was only able to correctly respond in $19.8 \%$ of the cases. (In a similar study only $21.3 \%$ of successful recognition was achieved [19]).

There were 4 types of errors: error in sound detection error (due to other ambient sounds, the system failed to detect the start of utterance) (17.3\%), ASR resulted in low reliability score (30.9\%), utterance did not match with the prepared grammar/vocabulary $(2.47 \%)$, and misrecognition in ASR (29.6\%). In case the mis-recognition occurred, often the system seemed to be interfered by ambient noise, which was matched with some vocabulary in the lexicon.

In contrast, in case ASR successfully detected the names, nicknames, or requestable properties, the system provided appropriate answers. Overall, this preliminary test revealed that the system is capable of handling users' utterances when the ASR is successful, while we would yet need to wait ASR technologies to be ready for real world environments.

\subsubsection{Wizard-of-Oz}

The system is ready for autonomous speech recognition. But, for this study, to focus on other parts of interaction rather than working for errors in speech recognition, we used a human operator to only support speech recognition.

We strictly limit the task of the operator, and have him work like dumb ASR software described in the previous section. We did not allow the operator to add his knowledge. Like the output from the ASR, the operator only typed the words included in what the user said. For instance, to our knowledge, if a user asks for a "Place for lunch" but such wording is not in the system vocabulary, in previous studies Wizard-of-oz operators replaced such words to the ones system can handle, like "restaurant"; by doing so, system can work with very limited vocabulary 
406 and knowledge. Instead, with our system, a novice person who does not know the environment 407 (e.g. list of shops) can easily serve as an operator.

408

409

410

411

412

413

414

\section{Preliminary trials: Lack of 'Knowledge' for Interaction}

We conducted a preliminary study with the system reported in the previous section. We initially intended to supplement missing data and evaluate its performance. We found the system itself worked well (we will report in section 6.3); however, interaction failed in other parts we did not think about. That is, some visitors responded in an unexpected way. In short, until here, we focused on the 'information' aspect, which we found to be satisfyingly prepared, but we found a problem in 'interaction'.

Here, we report two typical cases of failures. From these cases of failures, with a trial-anderror approach, we seek the reason why interaction fails and seek for better pattern of interaction for the problem. Finally, we generate hypotheses about missing knowledge in interaction (to be reported in the next section).

\section{Case 1: Interaction did not start}

The initial version of the robot imitated the interaction of human information staff. It waited for the arrival of the visitors, and waited for them to make a request. This is what a human staff member does. The signboard showing 'information staff' on the chest of the robot was very visible, so we expected that every visitor would have common expectations as those investigated in section 3.2 .

However, frequently there are people who stay in front of the robot without saying anything. Figure 6 shows one of such cases. A man stopped in front of the robot, and the robot was ready to receive a request, orienting its body and head toward him; but, without talking to it, he moved to a side of the robot, and the robot followed. He moved back, and it followed again. Finally, he left after 30 seconds of silence.

\section{Case 2: Passive visitors}

Further, we noticed that the conversation got stuck when it asked for a request, even though the user initially spoke to the robot. For instance, Figure 7 shows a visitor who engaged in 
435

436

437

438

439

440

441

442

443

444

445

446

447

448

449

450

451

452

453

454

455

456

457

458

459

460

greeting, but came to be silent when prompted to ask request. She left after 5 seconds of silence after being prompted.

We interpreted that such people do not have concrete requests in their mind, thus they were stuck when asked to offer requests.

\section{Field Experiment}

For each case of problems found in the preliminary trial (reported in the previous section), we generated a hypothesis, and conducted an experiment to confirm our idea to supplement such weakness. The study protocol was approved by institutional review boards of Advanced Telecommunications Research Instituted International with reference number 14-502-2.

\subsection{Experiment 1}

\subsubsection{Hypothesis}

We initially replicated the way of interaction of human staff. That is, we make it clear that the robot is serving as information staff. Assuming that visitors would have the common expectations of what an information staff is, we let the robot wait for a visitor to make a request, and to prompt to request if not asked. However, this assumption can be not always true. Visitors may not share or are unsure about their expectations of the 'information robot' role. If this is the case, we can probably moderate the problem by letting the robot first explain its role (direction giving and recommendation). Thus, we made the following prediction:

Prediction 1: If the robot proactively explains its role as information staff, people will more frequently request information from it.

\subsubsection{Participants}

The study was conducted during weekends. The participants were visitors of the shopping mall, who are typically group of friends and families who come to the mall for leisure. The mall is big, and the layout is complicated, thus people are often in real needs of getting directions from someone. 
When a robot is placed on the mall, people sometimes stopped at the robot. We assumed that such people who stopped at the robot as the participants.

463

464

\subsubsection{Condition}

There are two conditions compared.

- With self-introduction: when a person stops, the robot starts self-introduction. It says, "Hello, I can provide directions and recommendations". Then, it prompts him/her to request "May I provide you some information?"

- Without self-introduction: when a person stops, the robot waits him/her to request without speaking to the user.

In both conditions, when a visitor requests it immediately moves into the information dialog. After 20 seconds of silence, the robot closed the interaction saying "bye-bye".

\subsubsection{Procedure}

The robot was placed at a square of the mall (Figure 4 right). We choose this location because visitors often arrive from the nearby escalator, and need direction giving around this location. The study was conducted during daytime on weekends. We prepared six pairs of 25-minutes time slots. For each pair, two conditions were assigned. Between the slots, we put 5-minutes break, so that visitors are not influenced by the adjacent time slot.

The visitors of the mall were able to freely interact with the robot. There was a signboard showing 'information staff' on the frontal side of the robot, which was clearly visible to the visitors. Beyond that, there were no restrictions nor instructions provided to visitors. There was a person ensuring safety, but he stayed behind a column so that his presence was hardly noticeable from pedestrians. In such circumstances, we observed pedestrians' natural reaction to the robot.

\subsubsection{Measurement}

Considering the role of the information staff, we define the success of the interaction as follows:

$$
\underline{\text { Success: }} \text { The case where the robot was able to receive a request and offered appropriate }
$$
information/service.

We coded the success from the recorded video. Note that we only evaluated people who stopped in front of the robot (more than 3 seconds) and faced toward the robot; we consider that 
489 letting people stop is beyond the scope of this paper. If the same person interacted multiple 490 times, only the first one was evaluated. Further, we only evaluated one participant per group (i.e. 491 only the first member of the group, who stopped and faced the robot, was counted as our 492 participant), so that the experiment would not suffer from other members' prior interaction.

\section{6.1.6 Result}

494 In total, there were 238 interactions evaluated, which were coded by two coders who do not 495 know the study hypothesis. One coded the whole data and second one did confirmatory coding 496 for $10 \%$ of the data. Their coding results matches well (kappa coefficient .962).

497 Figure 8 shows the result of the study. There were $69.0 \%$ of the successful interactions in 498 the with self-introduction condition, while $54.4 \%$ in the without self-introduction condition.

499 Typical failure was, like the one shown in figure 6, where visitors stayed in front of the robot but 500 remained silent even if they were prompted to talk to the robot. Some visitors left in the middle 501 of the conversation, and some explicitly said they did not need service (6 cases in with self502 introduction condition).

503 significant difference between the conditions $(\chi 2(1)=4.755, \mathrm{p}<.05, \varphi c=.141)$.

506 Thus, the prediction 1 was confirmed. When the robot provides self-introduction, the interactions ended with success more frequently. We interpret that even though the robot serves an 'information' role, people should share a common expectation. Unless it explains its role, some people might fail in using it.

\subsubsection{Discussion}

511 It is plausible that there are two sources of failure addressed. One is the belief that the robot can 512 talk to them; another is the expectation that it offers information. We mainly argued the second 513 point, but it simultaneously offered help for the first element. Thus, one would argue that it is 514 better to compare with a robot that only speaks to users but does not provide self-introduction.

515 However, it was not easy to prepare such a condition, where the robot only shows the 516 capability that it can talk in the context of information service. For instance, if it only greeted 517 people, visitors might expect it to engage in variety of interactions, but in reality the robot can 
518 only react for the 'information' role. Thus, although the effect would be due to both elements, we

519 conducted the study in such a way. It remains as an open question what is the best length of self-

520 introduction. We could make it short and only imply its task by saying something like "may I

521 help you?" We consider that to our observation, people did not get bored due to length of the

522 self-introduction and thus it could be considered as reasonable.

\section{$523 \quad 6.2$ Experiment 2}

\subsubsection{Hypothesis}

525

526

527

528

529

530

531

532

533

534

535

536

537

538

539

540

541

542

543

544

545

In the experiment 1, we found that self-introduction moderated the problem of failure; yet, interaction failed for about $30 \%$ of the visitors. We hypothesized that there are visitors who initiated interaction out of curiosity, without a concrete request in mind. Such people would be stuck when a robot prompts them for a request in a direct way. We hypothesized that we can moderate this problem, if the robot turns its offer into a question that they can easily answer. Thus, we made the following prediction:

Prediction 2: If the robot prompts a user for a request in a way of questions they can easily answer, people will more frequently make requests to the information robot.

\subsubsection{Participants}

The same procedure was used as in experiment 1.

\subsubsection{Condition}

There are two conditions compared. In both conditions, when a person stops, the robot starts with a self-introduction, saying "Hello, I can provide directions and recommendations." This is identical to the wording used in experiment 1. After a short pause, the robot utters "I will give recommendations based on the locations you are going to", and prompts the user to ask. The prompting utterance differs depending on the following condition:

- Open-ended prompting: It prompts the user saying "What kind of recommendation do you wish?"

- Close-ended prompting: It prompts the user saying "Where are you going?" In both conditions, whenever a visitor requests something to the robot, it immediately moves into the information dialog. If the user keeps silent for 8 seconds, it once repeats the 
546 prompting utterance. If there were 20 seconds of silence after the prompting utterance, the robot

547 closed the interaction saying "bye-bye".

548

549 The same procedure was used as in experiment 1 . We prepared seven pairs of 25 -minutes time 550 slots.

551

552 The same measurement was used as in experiment 1.

\subsubsection{Result}

554 In total, there were 205 interactions evaluated, which were coded by two coders who do not 555 know the study hypothesis. One coded the all data and second one did confirmatory coding for $55610 \%$ of the data. Their coding results matches well (kappa coefficient .936).

557 Figure 9 shows the result of the study. There were $84.5 \%$ of successful interactions in close558 ended prompting condition and $69.4 \%$ in open-ended prompting condition. Similar to the 559 experiment 1 , in failure cases, some visitors kept silent when prompted, some visitors left in the 560 middle, and some explicitly said they did not need the service (3 cases in close-ended prompting 561 condition).

562 We applied a Chi-square test to evaluate the ratio of success against failures. There is a 563 significant difference between the conditions $\left(\chi^{2}(1)=5.678, p<.05, \varphi_{c}=.166\right)$.

564 The prediction 2 was consequently confirmed. When the robot's prompting was close565 ended, the interaction was more frequently successful than open-ended prompting. We interpret that as predicted many visitors did not have requests in mind and got stuck when asked to request; instead, if the robot offered a prompting utterance that invited the user to talk about what they know (e.g. their destination), it will more easily continue the dialog and offer information 569 requested by the user. 
571

572 There are some open questions remaining. One would argue that those who kept silent are people 573 who did not want to 'hear' the information, thus they did not respond to 'hear' questions in 574 close-ended prompting. It is possible that they did not have that much will to spontaneously ask 575 the robot to provide information; nevertheless, in open-ended prompting condition, people who 576 were coded as success stayed until the robot finished providing information. One would also 577 argue that the robot could anyway give information even if visitors kept silent. This is possible, 578 and maybe the robot should do so for the remaining $15.5 \%$ of people. Our assumption is that it is 579 probably better if they hear information they requested, rather than randomly chosen 580 information. We could not fully clarify why the remaining $15.5 \%$ of people who kept quiet in 581 close-ended condition. We tried to interview such people, but they did not want to be 582 interviewed.

\section{$583 \quad 6.3$ Evaluation of System Performance}

584 Throughout the experiment 1 and 2, the robot was controlled with the system reported in section 585 586 587 588 589 590 591 592 593 594

595

596 597 598 599

4. In total, there were 435 requests made for the information robot. We analyzed how they were handled, and evaluated whether the robot's responses were correct.

$66.8 \%$ of the case requests were a name of location and $4.0 \%$ were a nickname. In the other cases, these requests were turned into requestable properties: there were $4.4 \%$ item name, $14.6 \%$ category, $7.2 \%$ feature, $2.5 \%$ people activity, and $0.4 \%$ people's state. In $78.6 \%$ of cases, the system provided direction-giving service, and $21.4 \%$ recommendation service.

The appropriateness was evaluated by coders who do not know the study hypothesis. They judged based on the following criteria:

Correct: the information the user requested is included and correct in the response from the robot.

For instance, when a user asked "are there Japanese restaurants?" the coder judged whether the robot provided the information about any Japanese restaurant (if any), and whether the provided information is correct. There coding results show moderate matching (kappa coefficient was .481). 
There were $96.6 \%$ of cases judged as correct. Incorrect cases were caused by the lack of

601

602

603

604

605

606

607

608

609

610

611

612

613

614

615

616

\section{Limitation}

618 The content of knowledge can be local to the specific environment, robot, language, culture, and

619 so on. The common sense about what the information service is would differ across cultures.

620 Thus, if our study results were to be applied somewhere else, although we believe that most of

621 the framework and structure of knowledge is pertinent, we would probably need to carefully

622 adjust the knowledge. For instance, it is plausible that people in other cultures would inquire

623 information with a different form. Knowledge about interaction would also differ. People in

624 other cultures can be more or less open, active, hesitate, and/or curious, thus the effectiveness of

625 such strategy can be different. 
626

627 We investigated the knowledge relevant to information robot. First, we confirmed that what

628 visitors expect for an information robot well overlapped with what human information staff do.

629 We developed a knowledge representation for information robot. Our field study confirmed the

630 knowledge representation was useful. When users requested, the robot was able to provide

631 information with $96.6 \%$ of success. However, it also revealed that many people did not behave

632 the way they interact with human staff. Our initial version of interaction flow only allowed

$63355.4 \%$ of success in providing information, while visitors in failure kept silent during the

634 interaction. Through our field experiments, we found that some people need the robot to provide

635 self-introduction about its role, and some people need close-ended prompting, i.e. letting users

636 talk about what they know to make a request, instead of letting them generate a request. Finally,

637 the robot was able to provide information for $84.5 \%$ of visitors. What we changed might be

638 subtle, yet it changed the results a lot.

\section{ACKNOWLEDGMENTS}

\section{REFERENCES}

642

643

644

645

646

647

648

649

650

651

652

653

654

655

656

657

[1] J. Cassell, T. Stocky, T. Bickmore, Y. Gao, Y. Nakano, K. Ryokai, D. Tversky, C. Vaucelle, and H. Vilh jálmsson, Mack: Media Lab Autonomous Conversational Kiosk, in Proc. of Imagina vol. 2, ed, 2002, pp. 12-15.

[2] S. Kopp, P. A. Tepper, K. Ferriman, K. Striegnitz and J. Cassell, Trading Spaces: How Humans and Humanoids Use Speech and Gesture to Give Directions, in Conversational Informatics: An Engineering Approach, T. Nishida ed., pp. 133-160, 2008.

[3] T. Ono, M. Imai and H. Ishiguro, A Model of Embodied Communications with Gestures between Humans and Robots, Annual Meeting of the Cognitive Science Society (CogSci2001), pp. 732-737, 2001.

[4] Y. Okuno, T. Kanda, M. Imai, H. Ishiguro and N. Hagita, Providing Route Directions: Design of Robot's Utterance, Gesture, and Timing, ACM/IEEE Int. Conf. on HumanRobot Interaction (HRI2009), pp. 53-60, 2009.

[5] S. Thrun, M. Bennewitz, W. Burgard, A.B. Cremers, F. Dellaert, D. Fox, D. Hahnel, C. Rosenberg, N. Roy, J. Schulte, and D. Schulz, Minerva: A Second-Generation Museum Tour-Guide Robot, IEEE Int. Conf. on Robotics and Automation (ICRA1999), pp. 1999-2005, 1999. 
658 [6] H.-M. Gross, H. Boehme, C. Schroeter, S. Mueller, A. Koenig, E. Einhorn, C. Martin, M.

659

660

661

662

663

664

665

666

667

668

669

670

671

672

673

674

675

676

677

678

679

680

681

682

683

684

685

686

687

688

689

690

691

692

693

694

695

696

697

698

Merten, and A. Bley, Toomas: Interactive Shopping Guide Robots in Everyday Use Final Implementation and Experiences from Long-Term Field Trials, IEEE/RSJ Int. Conf. on Intelligent Robots and Systems (IROS2009), pp. 2005-2012, 2009.

[7] T. Kanda, M. Shiomi, Z. Miyashita, H. Ishiguro and N. Hagita, An Affective Guide Robot in a Shopping Mall, ACM/IEEE Int. Conf. on Human-Robot Interaction (HRI2009), pp. 173-180, 2009.

[8] M.-P. Daniel, A. Tom, E. Manghi and M. Denis, Testing the Value of Route Directions through Navigational Performance, Spatial Cognition \& Computation, vol. 3, pp. 269289, 2003.

[9] Y. Morales, S. Satake, T. Kanda and N. Hagita, Modeling Environments from a Route Perspective, ACM/IEEE Int. Conf. on Human Robot Interaction (HRI2011), pp. 441448, 2011.

[10] T. Kollar, S. Tellex, D. Roy and N. Roy, Toward Understanding Natural Language Directions, ACM/IEEE Int. Conf. on Human-Robot Interaction (HRI2010), pp. 259266, 2010.

[11] C. L. Sidner, C. D. Kidd, C. Lee and N. Lesh, Where to Look: A Study of Human-Robot Engagement, Int. Conf. on Intelligent User Interfaces (IUI 2004), pp. 78-84, 2004.

[12] B. Mutlu, J. Forlizzi and J. Hodgins, A Storytelling Robot: Modeling and Evaluation of Human-Like Gaze Behavior, IEEE-RAS Int. Conf. on Humanoid Robots (Humanoids'06), pp. 518-523, 2006.

[13] C. L. Sidner, C. Lee and N. Lesh, Engagement by Looking: Behaviors for Robots When Collaborating with People, ed, 2003.

[14] C. Rich, B. Ponsler, A. Holroyd and C. L. Sidner, Recognizing Engagement in HumanRobot Interaction, ACM/IEEE Int. Conf. on Human-Robot Interaction (HRI2010), pp. 375-382, 2010.

[15] Y. Kobayashi, et al., Choosing Answerers by Observing Gaze Responses for Museum Guide Robots, ACM/IEEE Int. Conf. on Human-Robot Interaction (HRI2010), pp. 109-110, 2010.

[16] D. L. McGuinness and F. Van Harmelen, Owl Web Ontology Language Overview, W3C recommendation, 2004.

[17] D. Brscic, T. Kanda, T. Ikeda and T. Miyashita, Person Tracking in Large Public Spaces Using 3d Range Sensors, IEEE Transaction on Human-Machine Systems, vol. 43, pp. $522-534,2013$.

[18] D. Fox, W. Burgard and S. Thrun, Markov Localization for Mobile Robots in Dynamic Environments, Journal of Artificial Intelligence Research, vol. 11, pp. 391-427, 1999.

[19] S. Matsuda, T. Jitsuhiro, K. Markov and S. Nakamura, Atr Parallel Decoding Based Speech Recognition System Robust to Noise and Speaking Styles, IEICE TRANSACTIONS on Information and Systems, vol. E89-D, pp. 989-997, 2006. 


\section{Table $\mathbf{1}$ (on next page)}

The analysis result of expectation for information 
2 Table 1 The analysis result of expectation for information

\begin{tabular}{|l|c|}
\hline \multicolumn{1}{|c|}{ Expectation } & Ratio \\
\hline Direction giving & $95.2 \%$ \\
Recommendation & \\
(inquiry) & $76.2 \%$ \\
Other & \\
Playing with children & $23.8 \%$ \\
Lost child & $4.8 \%$ \\
\hline
\end{tabular}

3 


\section{Table 2 (on next page)}

Possible relationships 
2 Table 2 Possible relationships

\begin{tabular}{|l|l|}
\hline Users' request & Possible relation \\
\hline \hline Item name & is sold at / is served at / is at \\
Category & belongs to \\
Features & is a feature of \\
People activity & is possible at \\
People's state & is satisfied/healed/solved at \\
\hline
\end{tabular}

3 


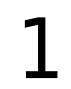

Information service
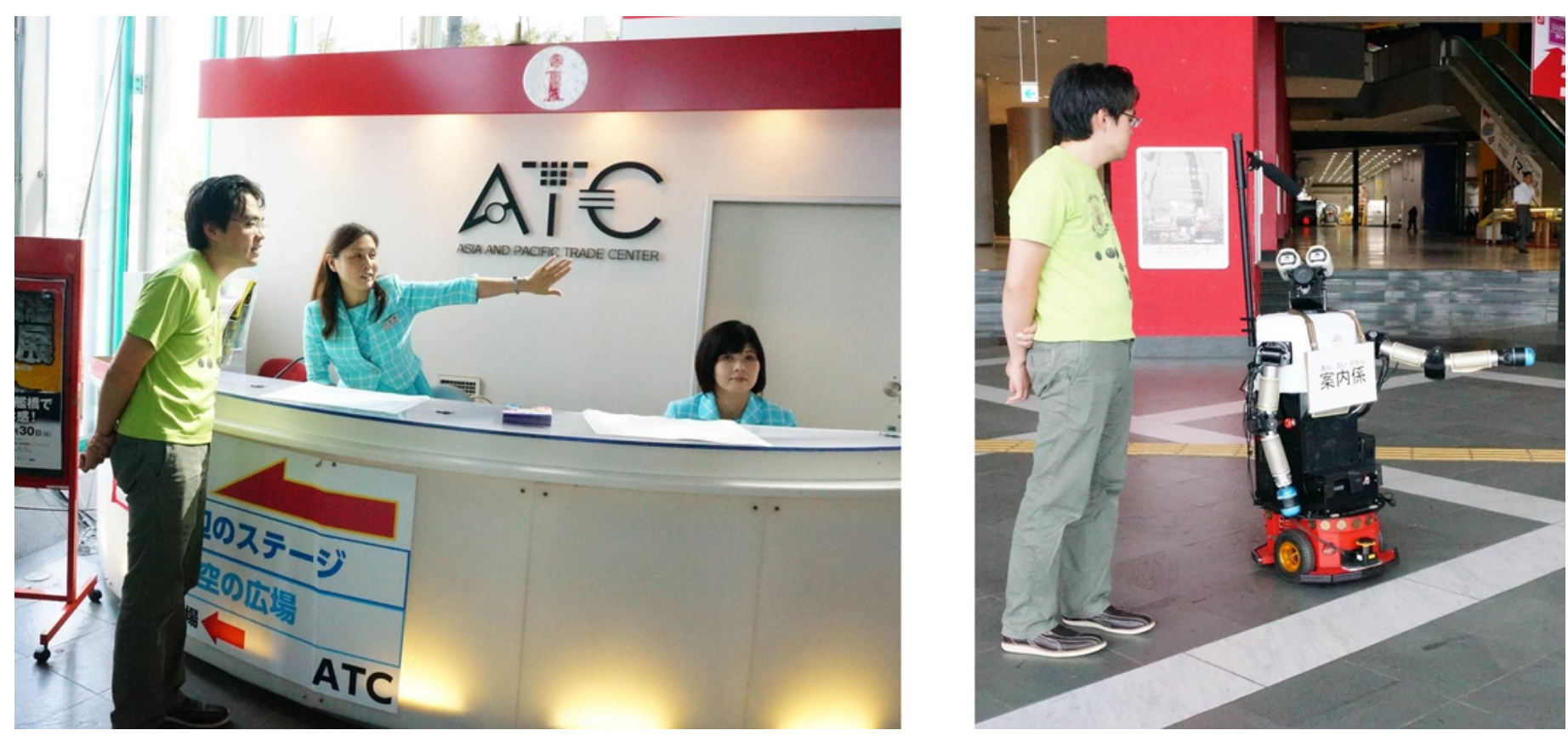
2

System architecture

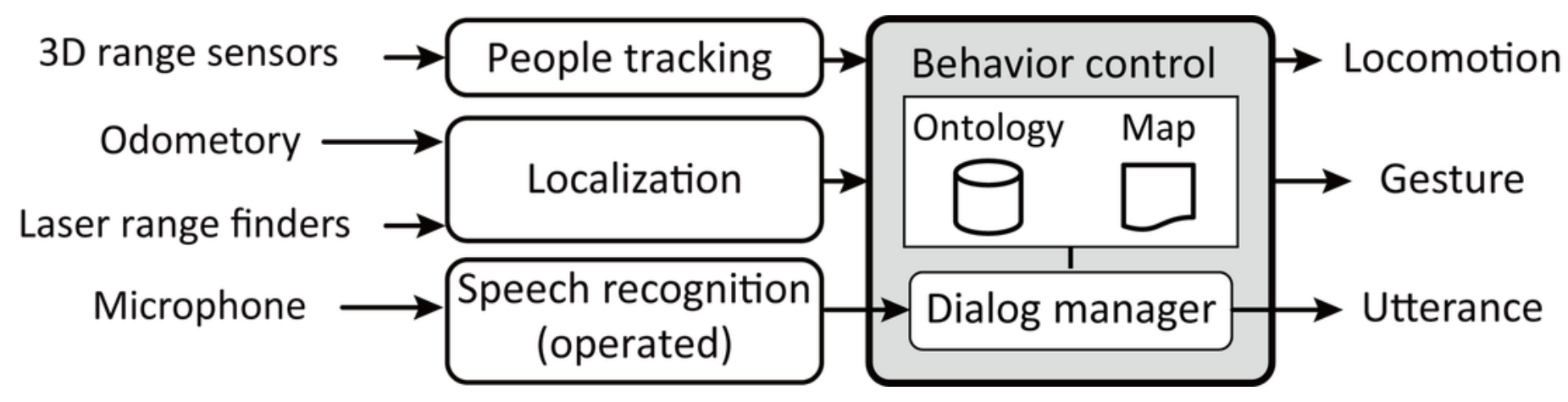




\section{3}

Environment of the shopping mall
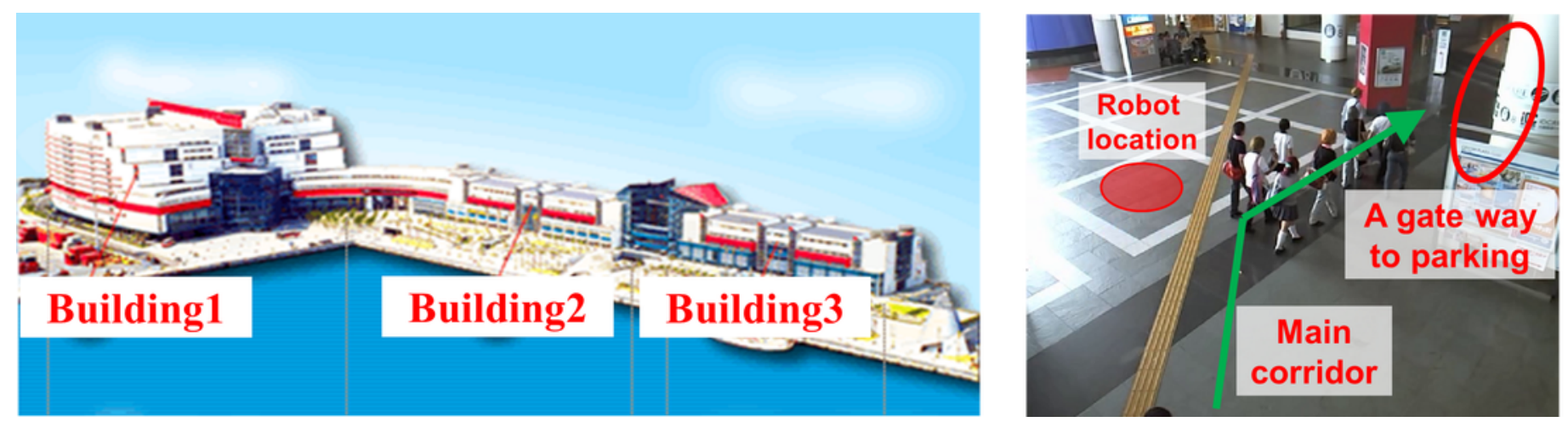
4

Knowledge representation for the environment

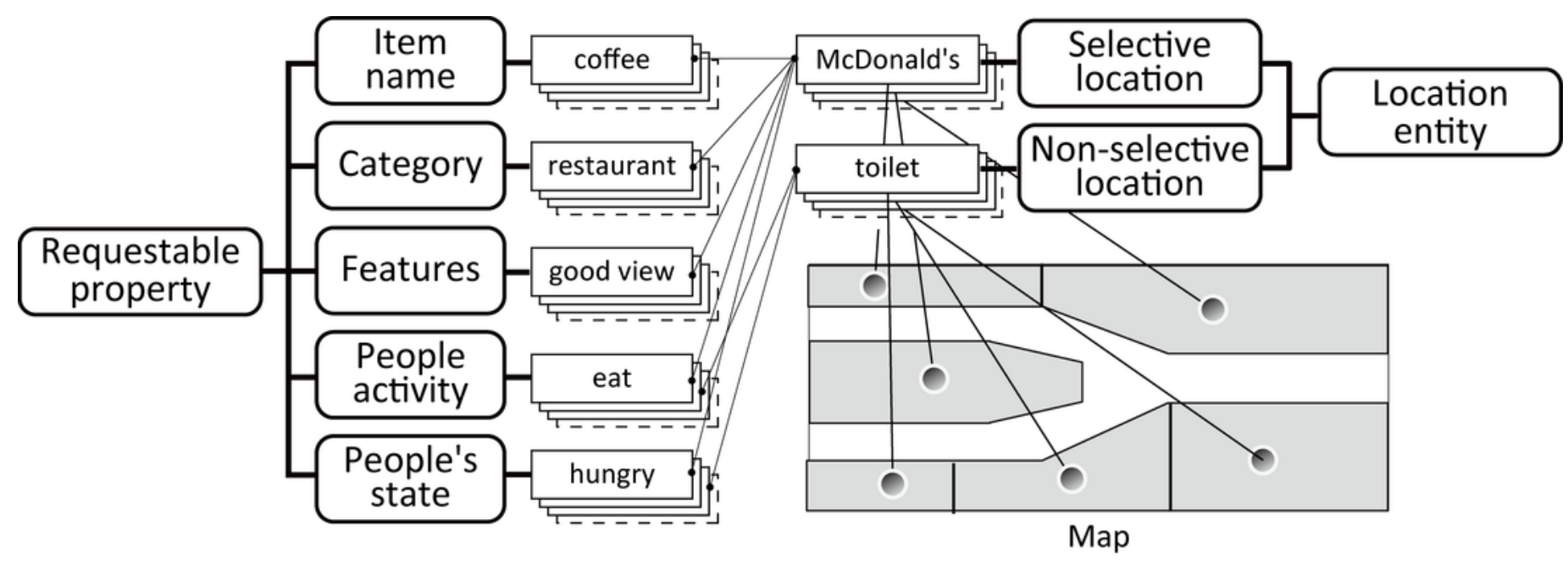


5

Illustration of the route perspective map

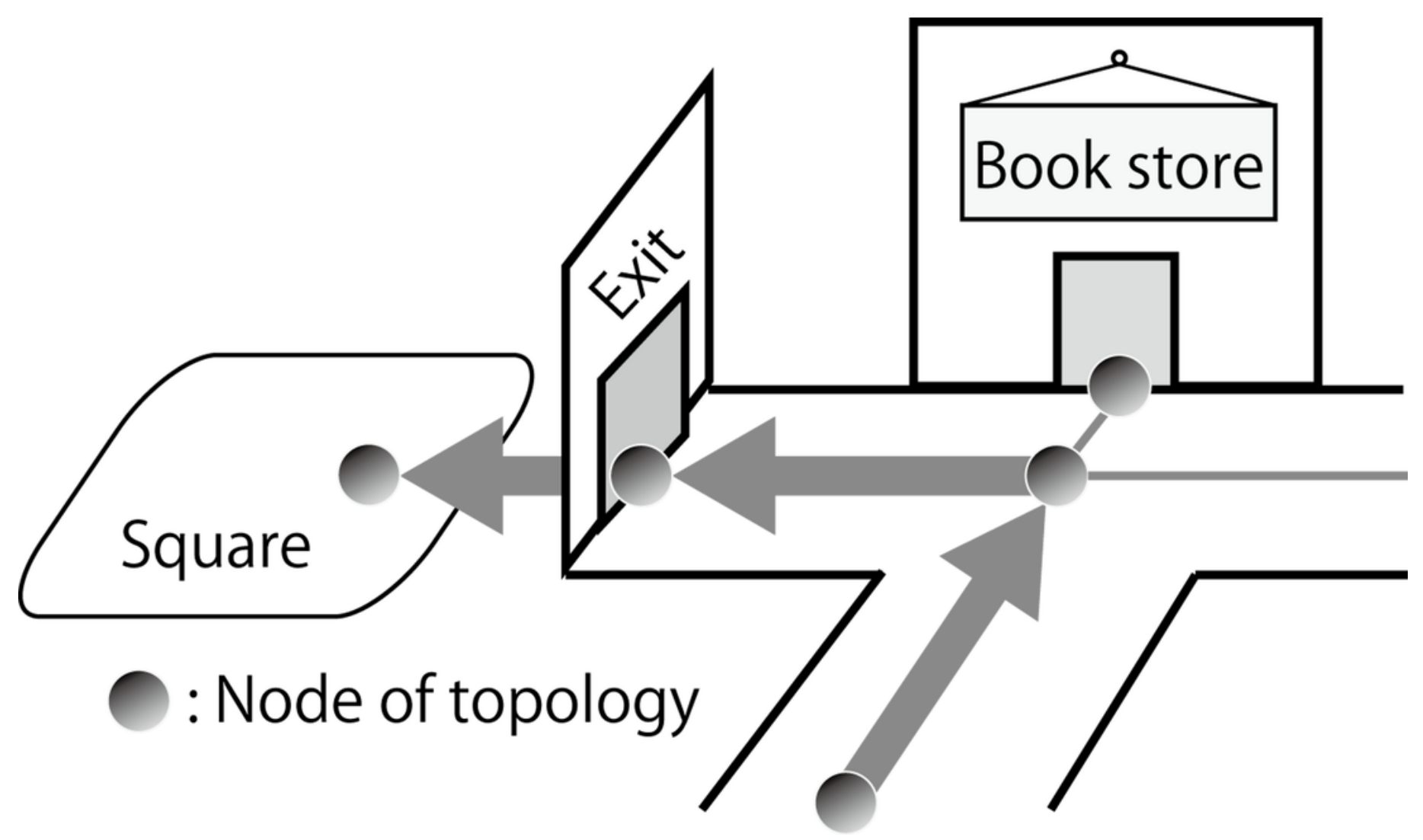




\section{6}

The interaction failed because the visitor did not speak
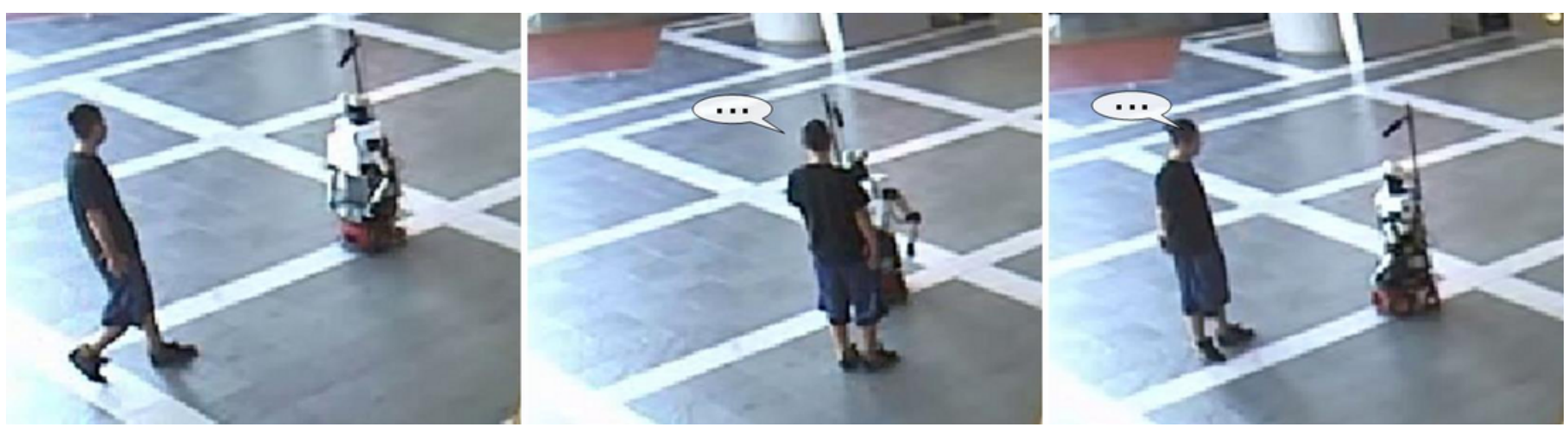


\section{7}

The visitor kept silent after prompted
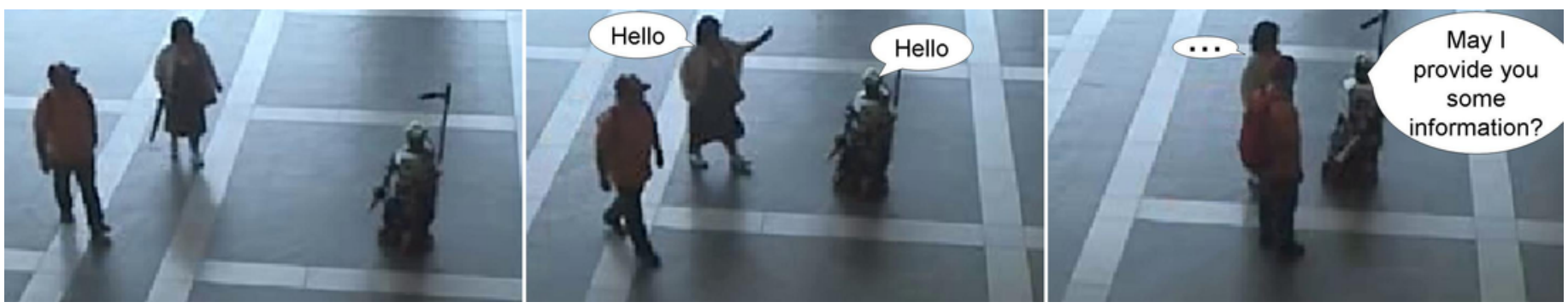
8

Result of the experiment 1

$$
p<.05
$$

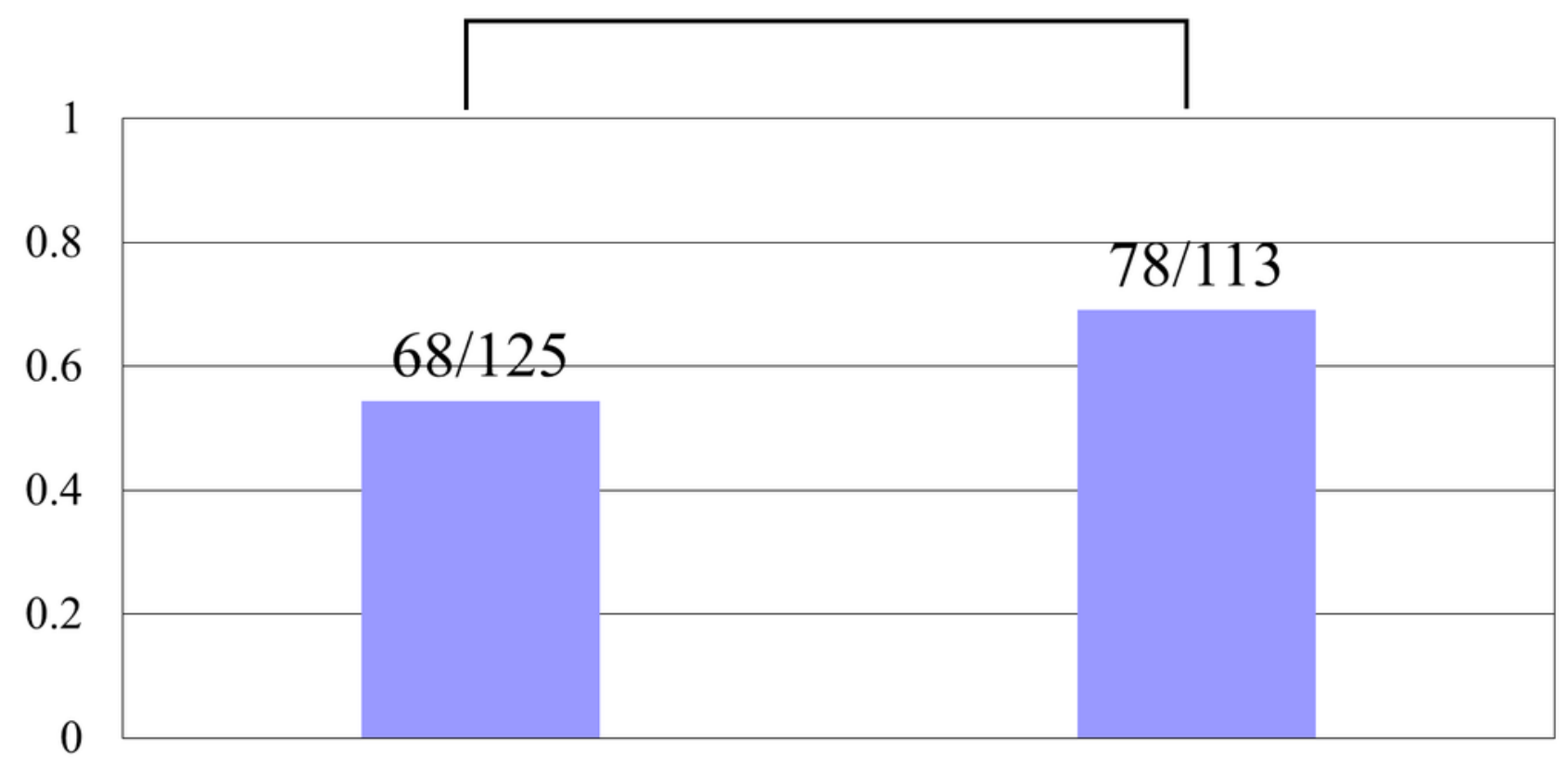

Without-self-introduction With-Self-introduction 
9

Result of the experiment 2

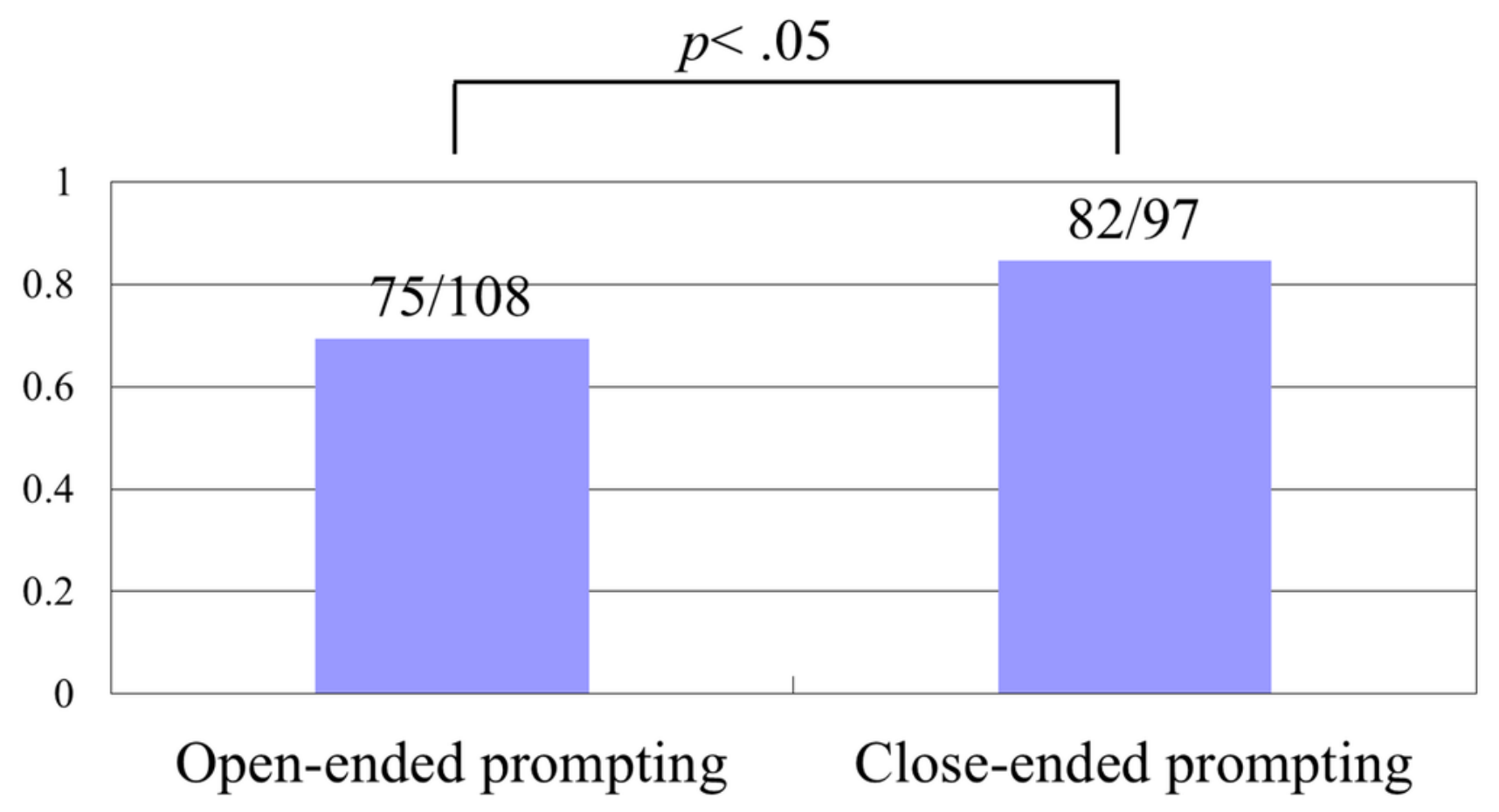




\section{0}

A scene of correct and successful interaction

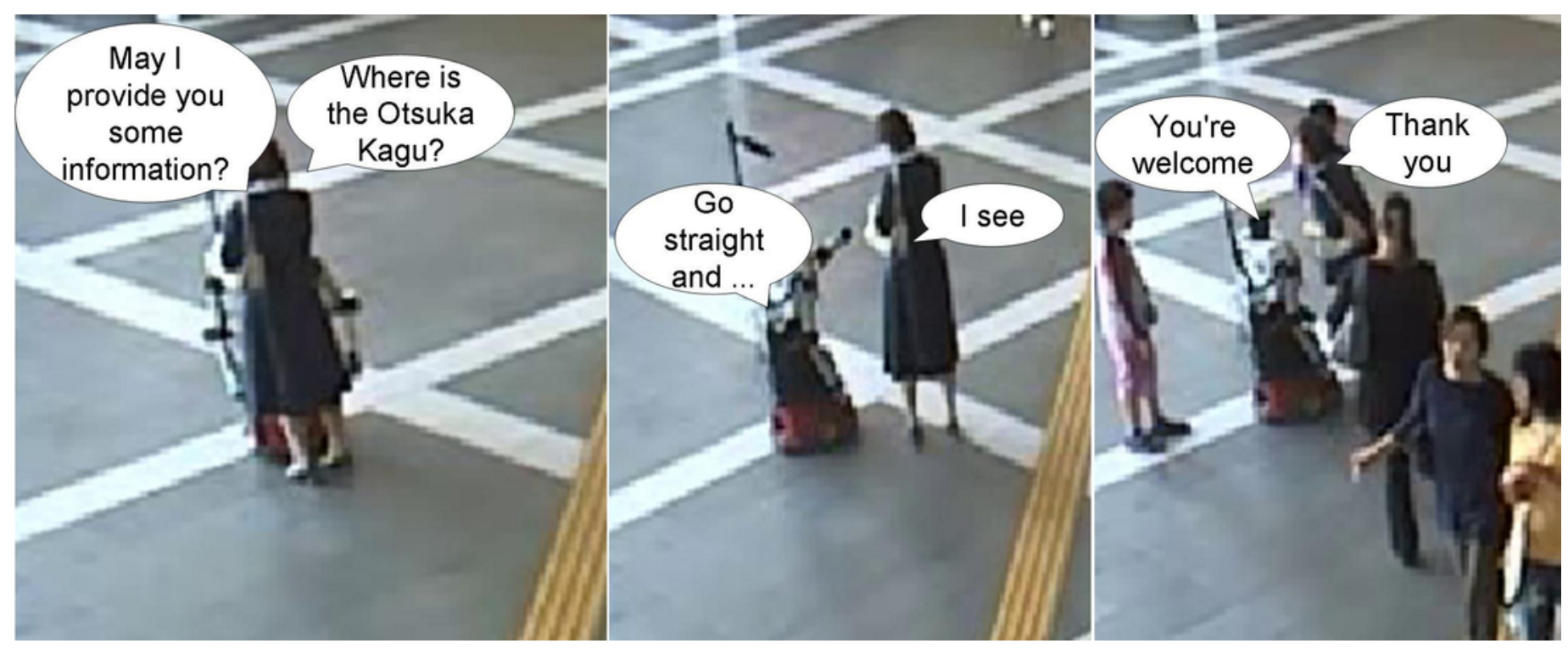




\section{1}

Request made based on visitors' state
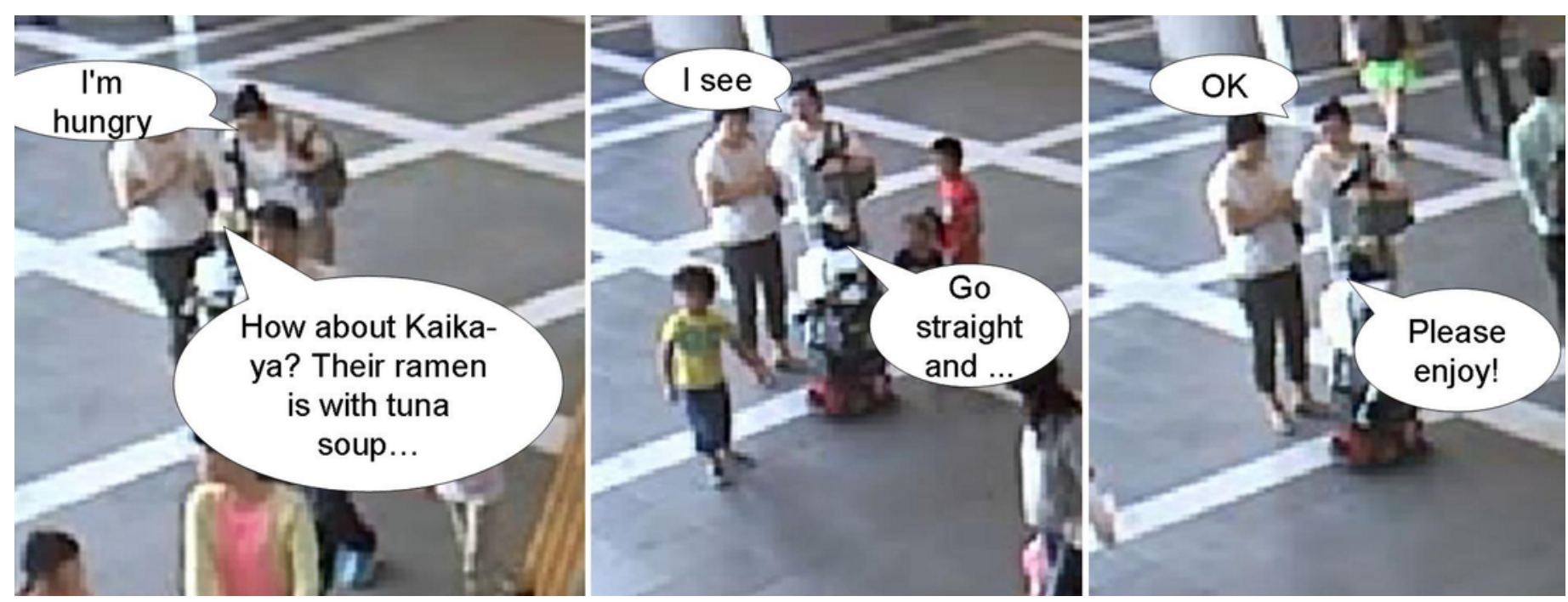